\title{
LA TÉCNICA DEL TAPIAL EN LA COMUNIDAD AUTÓNOMA DE MADRID. APLICACIÓN DE NUEVOS MATERIALES PARA LA CONSOLIDACIÓN DE MUROS DE TAPIA
}

\author{
(THE TECHNIC OF TAPIAL IN THE AUTONOMOUS COMUNITY OF MADRID USE OF NEW \\ MATERIALS TO CONSOLIDATE RAMMED EARTH WALLS)
}

Luis Maldonado Ramos y Francisco J. Castilla Pascual, Arquitectos; Fernando Vela Cossío, Arqueólogo Dpto. de Construcción y Tecnología Arquitectónicas (U.P.M.) Madrid

ESPAÑA

Fecha de recepción: $29-X-97$ $650-2$

\begin{abstract}
RESUMEN
La Comunidad Autónoma de Madrid, especialmente en su mitad meridional, conserva un amplio patrimonio de edificios construidos con tierra, con ejemplos de distintas técnicas como la tapia, adobe o los entramados de madera rellenos. La necesidad de conservación y mantenimiento de este patrimonio justifica la conveniencia de investigaciones sobre nuevas técnicas y procedimientos constructivos aplicables en trabajos de restauración y rehabilitación.
\end{abstract}

En este articulo se pretende dar una visión general sobre las particularidades de la técnica del tapial, predominante en nuestra región, y exponer los estudios realizados sobre el patrimonio existente asi como los objetivos, metodología y resultado de la investigación desarrollada para analizar la aplicación de nuevos productos en la consolidación de muros de tapia, realizada conjuntamente por el Departamento de Construcción y Tecnología Arquitectónicas de la Escuela Técnica Superior de Arquitectura de Madrid y la ONGD Inter-Acción.

\section{SUMMARY}

The use of earth as a building material is spread in a great part of the architectural heritage of the Autonomous Comunity of Madrid, specially in the southern region, it appears in different usages and building methods like rammed earth, adobe or filled wood frame structures. The need of maintenance and preservation of this heritage justify the convenience of research on new technics and materials appliable to restoration works.

This paper aims to offer a view on the particularities of rammed earth (tapial), predominant in this region, and show the studies carried out on the architectural heritage as well as the targets, methodology and results of the research works developed to analize the use of new products to consolidate rammed earth walls. The work has been carried out conjunctly by members of the Department of Construction of the Politechnic University of Madrid and the ONGD Interaction,

\section{LA CONSTRUCCIÓN CON TIERRA EN LA COMUNIDAD DE MADRID}

El uso de la tierra como material de construcción se encuentra extendido en buena parte de nuestra región, observándose diversas aplicaciones y sistemas constructivos. El área geográfica con mayor abundancia es la mitad meridional de la provincia de Madrid, correspondiente a los valles de los ríos Tajuña, Henares y Jarama: Mientras que en la zona occidental se encuentran mayoritariamente muros de entramado de madera y plementería de adobe, en la zona oriental se detectan principalmente sistemas constructivos de muros de tierra apisonada o tapia (1).

\subsection{Catalogación de sistemas constructivos}

En una primera fase se realizó un estudio cuyo objetivo era la documentación y el análisis del Patrimonio arquitectónico construido con tierra en la Comunidad de Madrid para la transferencia de los resultados obtenidos a ta Dirección General de Arquitectura y a la de Patrimonio Histórico Inmueble del gobierno regional. 
Para el desarrollo de este trabajo se ha contado con la colaboración de los alumnos de último curso de carrera de la Escuela Técnica Superior de Arquitectura de Madrid, participantes en el Seminario de Construcción con tierra, en el que se han incluido diversas conferencias de apoyo para la realización del mismo. Se formaron 24 equipos sobre un total de 67 alumnos, organizándose el trabajo en dos fases, una primera fase de trabajo de campo y toma de datos en los municipios y una segunda fase de elaboración de ejercicios prácticos, bien sobre un determinado sistema constructivo, sus características, patología y procedimientos de intervención, bien sobre un determinado edificio o elemento, con análisis de su estado de conservación, características constructivas y propuestas de rehabilitación concretas (2).

Para las labores de toma de datos se confeccionaron unas fichas que permitieron recoger, de manera sistematizada, datos generales de edificios y de las distintas técnicas constructivas que en ellos se observaban. Nuestro estudio se centra en aquellos ejemplos en que predomina la técnica del tapial (3), es decir, en la ejecución de muros o tapias mediante el apisonado de material (básicamente tierra) en sucesivas capas o tongadas dentro de un encofrado de madera de dimensiones adecuadas para ser trasladado progresivamente. Esto ha permitido obtener unos primeros resultados sobre las diferencias en la aplicación del sistema, materiales empleados, soluciones constructivas y patología, que a continuación se exponen.

\subsection{Técnicas de construcción con tapial}

Básicamente hemos diferenciado dos categorías de tapias o fábricas: tapias monolíticas, en las que el muro se construye de manera homogénea, existiendo variedades en función de la composición de la tierra y materiales apisonados o el tratamiento de las juntas, y tapias mixtas, en las que aparecen elementos verticales (machos $o$ machones) compuestos por diferentes materiales no apisonados, embutidos en el muro como refuerzo.

En el primer caso, podemos encontrar soluciones de:

.Tapia con tierra en estado natural, en la que tan sólo se produce un cribado, aireado y humedecimiento de la misma previo a su compactación.

.Tapia de tierra mejorada con grava, cascotes u otros materiales de forma quegaranticen una mejor compactación y aumento de resistencia. Una de las soluciones tradicionales es la adición de cal mezclada con la tierra conocida como tapia real.

.Tapia reforzada en las caras, donde a diferencia del caso anterior, los refuerzos (normalmente piedras) no se mezclan sino que se disponen junto a las caras del tapial antes del apisonado de cada tongada, permitiendo una mejor (c) Consejo Superior de Investigaciones Científicas Licencia Creative Commons 3.0 España (by-nc) adherencia del revoco (Fig.1). Técnicas similares son aquéllas en las que el careado se ejecuta con ladrillos (característico de las tapias valencianas) o con pelladas de cal (calicostrado).

.Tapia con juntas reforzadas que evitan el agrietamiento de las mismas por efecto de la retracción que se produce durante el secado de la fábrica. Generalmente estos refuerzos se realizan con morteros de cal o yeso.

.Tapia con juntas reforzadas curvas o brencas (4) que parecen emplearse para evitar el apisonado en los rincones del tapial donde reviste mayor dificultad la compactación de la tierra (Fig.2). Es frecuente encontrar soluciones en que las juntas se disponen alineadas verticalmente en vez de contrapeadas (Fig.3).

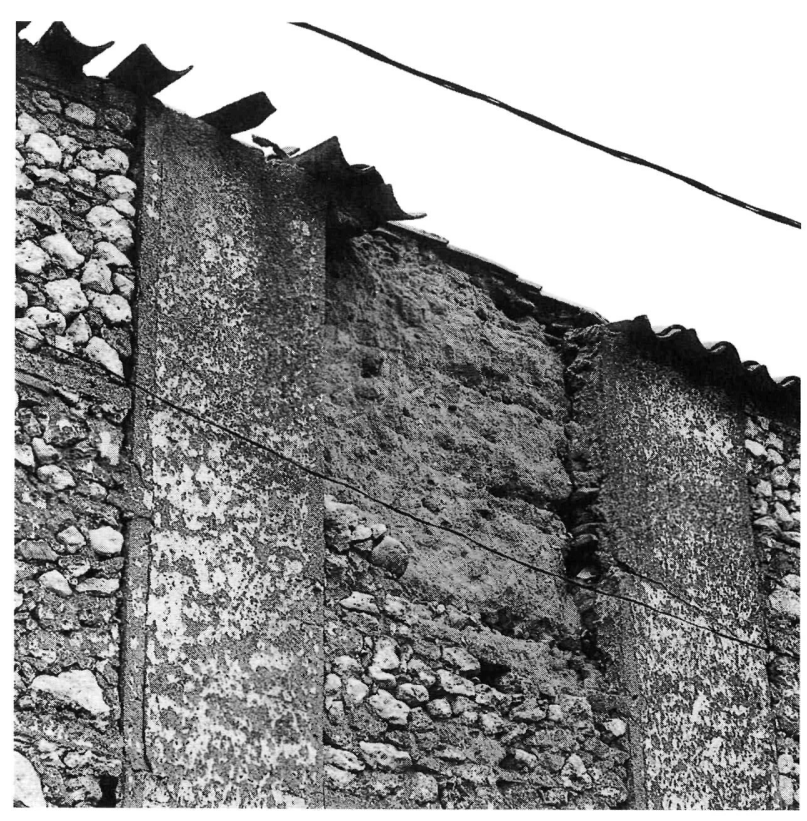

Fig. I.- Tapia con caras reforzadas (Loeche's).

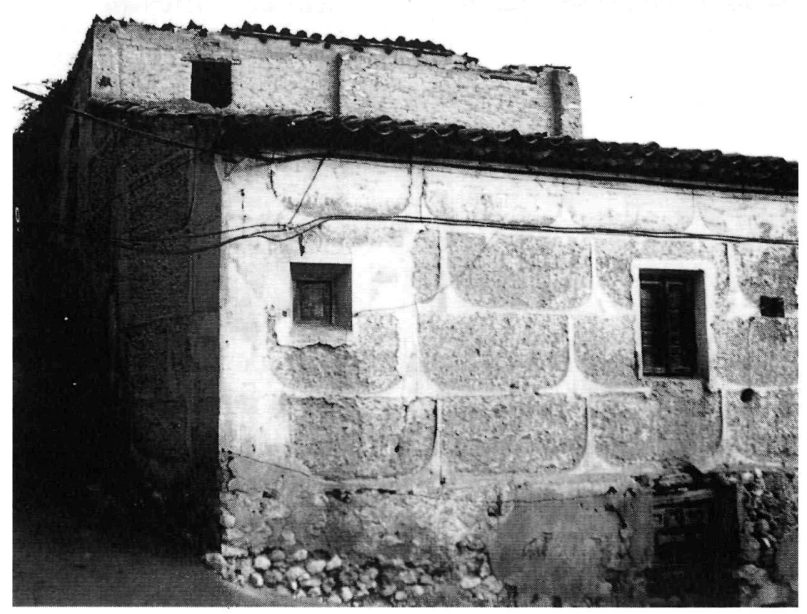

Fig. 2.- Vivienda en Perales de Tajuña.

http://informesdelaconstruccion.revistas.csic.es 


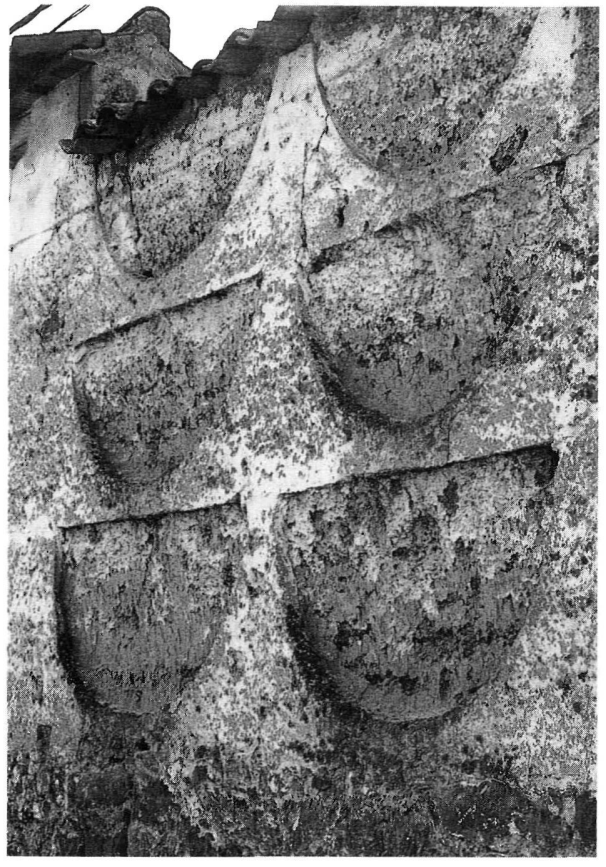

Fig. 3.- Tapia con "brencas" en Casarrubuelos.

En el segundo caso, distinguiremos también varios tipos:

.Tapias con machones, consistentes en rellenar mediante tapias el espacio comprendido entre dos elementos verticales de fábrica de ladrillo, adobe o yeso (Fig.4). Estos machones pueden recibir las cargas principales del sistema estructural horizontal, aunque, a menudo, el conjunto trabaja como un muro de carga homogéneo. Es corriente que las tapias con machones de ladrillo presenten igualmente las juntas horizontales reforzadas con una $o$ varias verdugadas del mismo material. Los machones de yeso suelen estar formados por mampuestos o cascotes y un mortero de este material.

.Tapias con rafas, o machones curvilíneos resultantes de combinar la solución anterior con las brencas (Fig.5).

.Tapias con entramado de madera, técnica poco corriente, directamente relacionada con los sistemas de entramados y plementería de adobe más comunes en la zona serrana y en la submeseta Norte. El espesor de las fábricas es mucho menor, empleándose en medianeras y cerramientos de desvanes.

\subsection{Soluciones constructivas}

Después de documentar los diferentes tipos de tapia detectados, se hizo un análisis de las soluciones constructivas más frecuentes en los puntos singulares de la edificación. El sistema estructural habitual en las construcciones de tierra es el de muros de carga. Las cimentaciones tienen, en la mayoría de los casos, el del propio muro de tierra o algo más, oscilando entre 60 y $80 \mathrm{~cm}$. Se construyen casi (c) Consejo Superior de Investigaciones Científicas Licencia Creative Commons 3.0 España (by-nc)

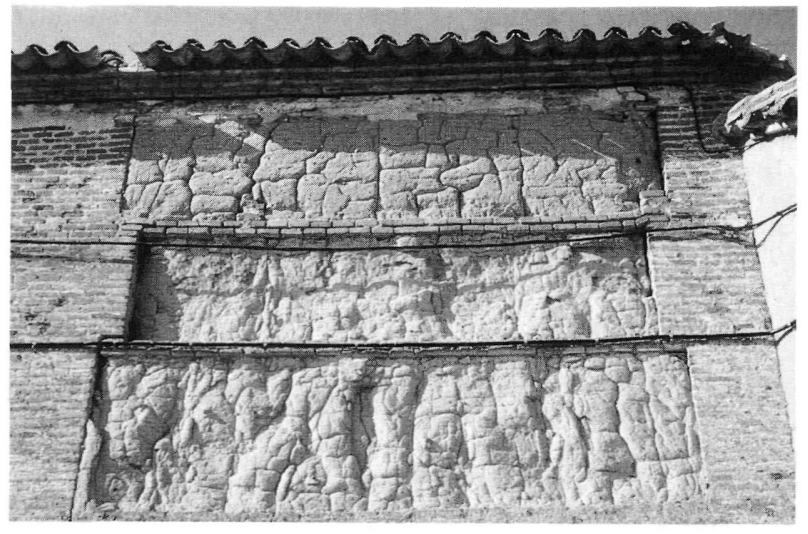

Fig. 4.- Muro de tapia con machones de ladrillo (Valdeavero).

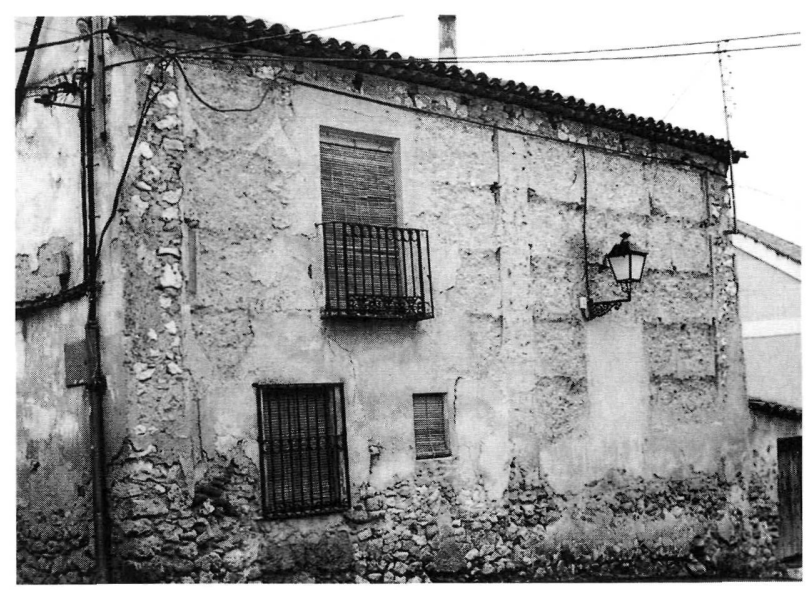

Fig. 5.- Vivienda en Chinchón.

siempre con mampostería, en seco, recibida con mortero de cal o con la misma tierra que forma el resto del muro. A los mampuestos que componen el muro se le añaden a veces cascotes, trozos de teja o de ladrillo.

Los muretes de cimentación se levantan, habitualmente, por encima del nivel del suelo a modo de sobrecimiento o zócalo. Los zócalos son un elemento fundamental en los muros de tierra, protegiendo el muro de las humedades de capilaridad. La altura del zócalo oscila normalmente entre 0,5 y 1 metro, aunque hay veces que abarca toda la planta baja e incluso más, relegando el muro de tierra al sobrado. En construcciones muy rústicas, como cercados o establos, se encuentran muros de tierra sin zócalo. Estos zócalos son, al igual que la cimentación, de mampostería, en la mayoría de los casos, aunque hay algunos de ladrillo en tapias, con machones de este material; en otros casos, el ladrillo se utiliza como remate de una mampostería a modo de verdugadas, para regularizar la altura y disponer una base horizontal sobre la que empezar a apisonar el material.

Las esquinas de un muro de tierra rara vez se dejan sin refuerzo, ya que, debido a su baja resistencia a impactos y erosiones, resultan un punto vulnerable. El caso más 


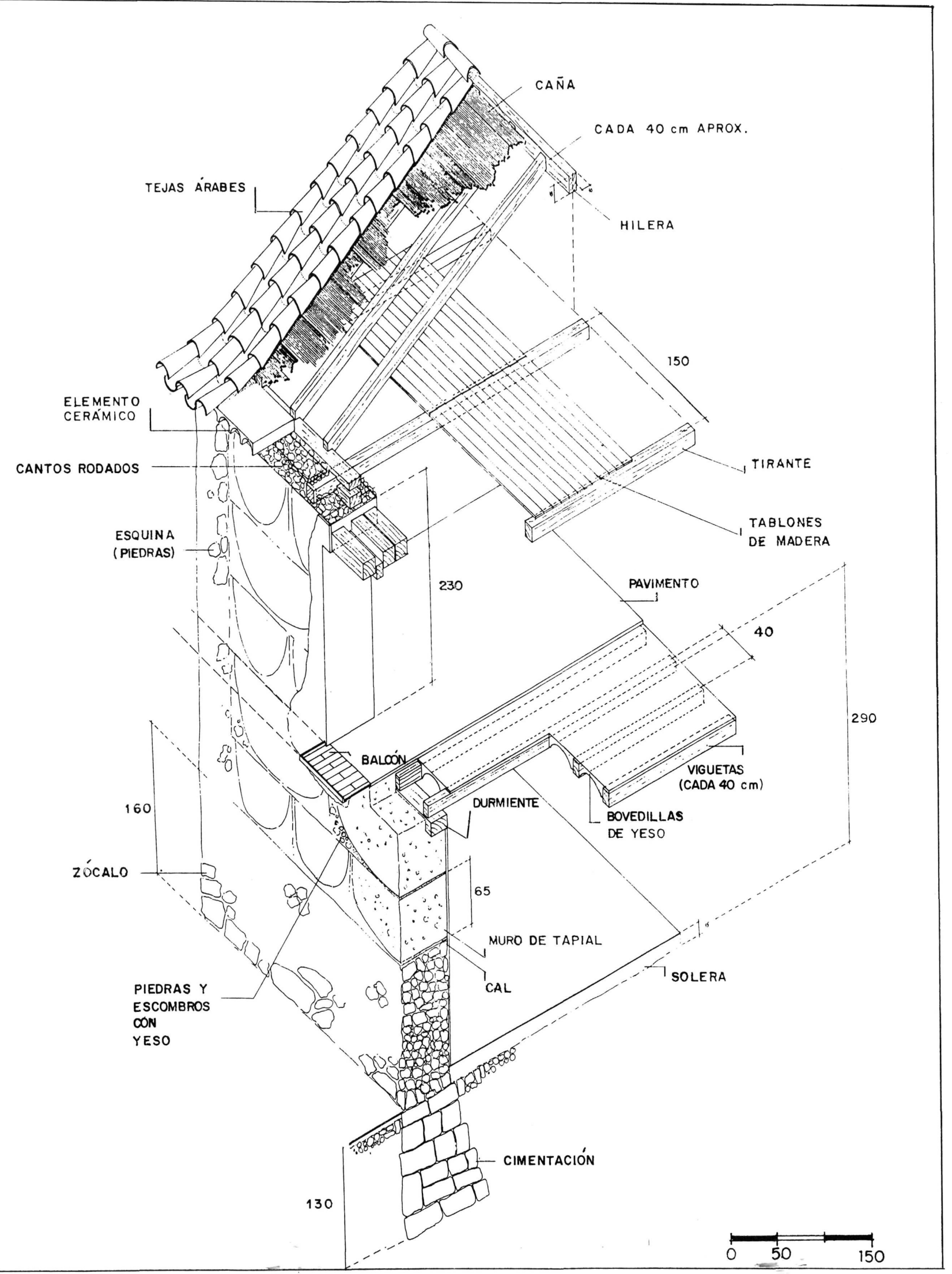

Sección constructiva de vivienda en Chinchón. Dibujo realizado por Gema Escalada y Mónica Megías, alumnas de último curso de carrera de la escuela Técnica Superior de Arquitectura de Madrid.

(c) Consejo Superior de Investigaciones Científicas Licencia Creative Commons 3.0 España (by-nc) 
frecuente era construir la esquina con mampostería, siguiendo el mismo sistema utilizado en cimentaciones, zócalos o machones, si existen. Encontramos así, esquinas de yeso, ladrillo e incluso de adobe, solución esta última que no parece la más adecuada. Un caso menos frecuente, es la esquina trabada con piezas de sillería, apreciable en edificios de cierta relevancia.

Los forjados son muy parecidos en todas las construcciones tradicionales: viguetas o rollizos de madera apoyando sobre los muros. Sobre estas viguetas se colocan tablas o revoltones de yeso rellenos con cascotes. El apoyo de estas viguetas sobre el muro de tierra supone un problema, debido a la baja resistencia de la tierra frente a cargas puntuales. Paraevitar unaexcesiva concentración de cargas, la solución más empleada consiste en colocar un durmiente de madera sobre el muro. En este durmiente apoyan las viguetas, quedando así la carga, uniformemente repartida. El durmiente se coloca en la cara interior del muro, protegiendo la madera de los cambios de humedad. En algunas ocasiones, este durmiente se sustituye por una capa de tierra mezclada con cal, que sirve igualmente para repartir las cargas y además ayuda a nivelar el plano de apoyo de las viguetas. En los muros de tapial con entramado de madera, es frecuente ver las viguetas del forjado encajadas entre un durmiente y una solera. El primero sirve de apoyo a las viguetas, la solera recibe los pies derechos que refuerzan el muro.

Los encuentros de muros y cubiertas plantean dos problemas: el primero, al igual que en los forjados, es resolver la transmisión del peso de la cubierta al muro sin cargar éste excesivamente. En el caso de la cubierta se complica más, ya que las cargas, al ser inclinadas, tienen una componente horizontal, siempre más difícil de transmitir al terreno. Para solucionar esto se colocan durmientes, al igual que en los forjados con estribos o tirantes, evitando, así, transmitir al muro estos esfuerzos horizontales. Otro problema es la solución adecuada del alero, que evite en lo posible filtraciones del agua que escurre por las cubiertas deteriorando la coronación del muro, para lo que se dan distintas posibilidades:

. Las pares de la cubierta vuelan sobre la fachada, soportando la cobertura.

. Colocación de piezas específicas de madera (canecillos), que se apoyan en el durmiente de coronación y vuelan sobre el muro.

. Hiladas de ladrillo o teja embutidas en el muro, que vuelan sucesivamente unas sobre otras.

Los huecos abiertos en muros de tierra aparecen siempre reforzados con materiales más resistentes, de-manera análoga a las soluciones de esquina. El material utilizado para estos refuerzos es muy variable y está relacionado con (c) Consejo Superior de Investigaciones Científicas Licencia Creative Commons 3.0 España (by-nc) la técnica empleada para construir el muro. En tapias monolíticas, lo más frecuente es colocar piezas de madera bordeando el hueco, situándose el cerco a haces exteriores del muro, protegiendo el alféizar interior. En tapias mixtas, se refuerzan las jambas con el mismo material que forma los machones. Otra solución menos frecuente consiste en bordear los huecos con sillería o con dinteles y jambas monolíticas de piedra.

En cuanto a los dinteles, son, en casi todos los casos, piezas de madera, a veces escuadrada y en otros casos, simplemente, rollizos. Es frecuente enrollar cuerdas sobre las piezas de madera, para mejorar la adherencia del revestimiento. Por este mismo motivo se encuentran también piezas de madera con hendiduras de azuela.

Es muy importante en los muros de tierra una buena protección superficial; tradicionalmente, el material de estos revestimientos era el mortero de barro, utilizando una tierra arcillosa para mejorar la impermeabilidad; esta tierra se mezclaba con paja, con el fin de mejorar la cohesión y evitar fisuras. Otros revestimientos frecuentes eran el revoco o el encalado sobre la propia fábrica (Fig.6).

\subsection{Patología}

Como parte final del estudio se hizo un análisis de los daños más frecuentes y característicos de esta clase de fábricas:

\subsubsection{Grietas}

Debido a la retracción que sufren estas fábricas durante el período de secado, es frecuente la separación de las juntas entre tapias en muros monolíticos o entre éstas y los machones en muros mixtos. Los machones (especialmente los de albañilería) presentan secciones variables en su altura, actuando como enjarje con la tapia y mejorando la cohesión entre ambos materiales. En la mayoría de los muros de tierra estudiados aparecen grietas alrededor de los huecos, bien siguiendo los bordes de éstos, o formando arcos de descarga sobre el dintel, aunque es dificil precisar si su alcance es estructural en el muro.

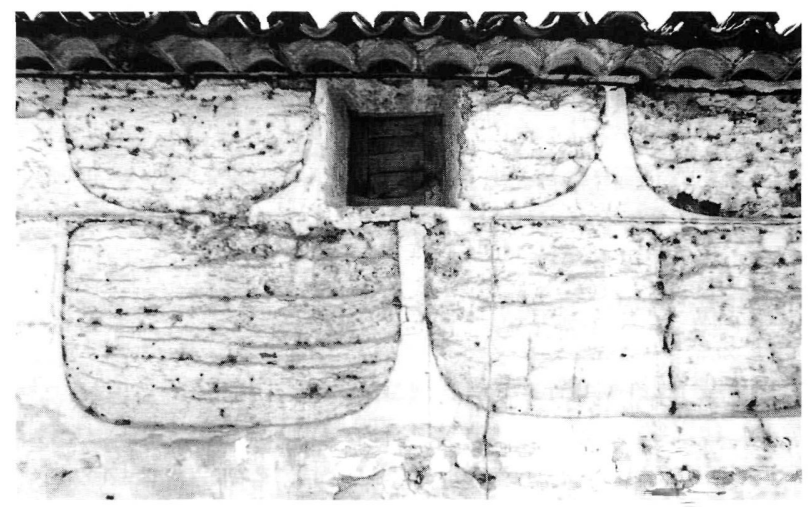

Fig. 6.- Muro erosionado en el que se aprecian sucesivas reparaciones del revestimiento. 


\subsubsection{Erosiones}

La tierra es un material con baja resistencia superficial frente a impactos y erosiones. Por esto es muy importante un adecuado revestimiento que proteja el grueso de la fábrica, ya sea proporcionado por el mismo proceso constructivo (calicastrado) o aplicado posteriormente. Cuando este revestimiento es poco resistente, o se pierde, los muros de tierra son muy erosionables por el viento, la lluvia y el ataque de insectos $u$ otros organismos que anidan fácilmente en los mismos. Es corriente observar una erosión marcada en franjas horizontales cuando el espesor de las tongadas es excesivo (superior a $10 \mathrm{~cm}$ ) produciéndose una compactación defectuosa en la parte inferior.

\subsubsection{Humedades}

Éste es uno de los problemas más frecuentes de los muros de tierra, debido a la poca impermeabilidad de este material. Distinguiremos dos tipos, fundamentalmente: las de capilaridad y las de filtración. Las primeras se manifiestan en las partes bajas del muro, formando una línea sensiblemente horizontal. La consecuencia más frecuente de estas humedades es el desprendimiento del revoco, al debilitarse la adherencia a la fábrica. Con frecuencia se ha intentado solucionar el problema colocando una capa de mortero de cemento sobre la zona afectada, pero con esto lo que se consigue es que el agua ascienda aún más y las manchas aparecen por encima del mortero. Las humedades de filtración, ya sea por absorción de agua de lluvia a través del muro o por fallos en la cubierta, debida a roturas de los aleros, provoca la disgregación del muro.

\subsubsection{Desprendimiento de revocos}

El desprendimiento del revoco puede ser causado por problemas del mismo (falta de adherencia, excesiva rigidez, etc.) y otras veces es el reflejo de un problema del muro sobre el que está aplicado. El revoco suele agrietarse en las juntas de un tapial mixto, debido a que la tierra se dilata más con la absorción de agua que el resto del muro, dando lugar a movimientos diferenciales. Los morteros de cemento, mucho más rígidos que el soporte sobre el que se aplican, se desprenden con mayor facilidad si no se han aplicado varias capas, aumentando progresivamente su dosificación

\section{TÉCNICAS DE INTERVENCIÓN PARA LA CONSOLIDACIÓN DE MUROS DE TAPIAL}

\subsection{Objetivos}

Como objetivo principal de esta investigación se planteaba la realización de un estudio piloto encaminado al desarrollo de una metodología específica, cuyos resultados se pudiesen aplicar a un proyecto de investigación más amplio, referente al Patrimonio Arquitectónico construido con tierra, de todo el Estado. Como objetivo específico se planteaba el desarrollo de nuevas técnicas de intervención en proyectos de restauración del Patrimonio Arquitectónico construido con la técnica del tapial. Para ello se experimentan los nuevos sistemas y materiales a emplear, con el fin de garantizar su perfecto funcionamiento cuando sean aplicados por las empresas constructoras.

\subsection{Metodología}

Con el programa de ensayos, quea continuación se describe, se pretendía alcanzar estos fines de dos maneras distintas:

-En primer lugar, realizando un análisis comparativo del comportamiento del material tierra aditivado con distintos productos hidrofugantes existentes en el mercado, para determinar cuál de ellos mostraba una mejor compatibilidad.

-En segundo lugar, comparando el comportamiento del material en probetas sometidas a ensayos de laboratorio con el de muros experimentales, para determinar, hasta qué punto, los resultados obtenidos en las primeras son extrapolables a las fábricas in situ

A partir del análisis documental y de los numerosos ejemplos estudiados en la fase anterior, se ha podido constatar, como principal agente erosivo de este tipo de fábricas, al agua, por lo que en el plan de ensayos priman aquéllos en que se somete al material a procesos en los que interviene este elemento, lo que permitió establecer una primera clasificación de productos según su comportamiento frente a la absorción de aguay heladicidad. La realización de ensayos de otra índole (resistencia o dureza) permitió contrastar si la aplicación de estos productos implica alteraciones importantes en la respuesta de las fábricas ante acciones mecánicas. Como se ha mencionado anteriormente, estos ensayos se realizaron paralelamente en probetas de laboratorio y en muros fabricados a la intemperie, utilizando como punto de partida el corpus teórico y documental de la tesis doctoral de Dña. Melín Nava Hung titulada: La arquitectura de tierra, su consolidación a través de la utilización de productos hidrofugantes: Experimentación sobre el centro histórico de Alcalá de Henares, dirigida por el Dr. Monjo Carrió y leída en nuestro Departamento, en 1995.

\section{Ensayos de laboratorio}

- Ensayo de humedad-sequedad (probetas $4 \times 4 \times 16 \mathrm{~cm}$ ). - Ensayo de hielo-deshielo (probetas cilíndricas, de $12 \mathrm{~cm}$ de diámetro $\times 15 \mathrm{~cm}$ de altura).

- Ensayo de compresión simple (probetas $7 \times 7 \times 7 \mathrm{~cm}$ ).

- Ensayo de goteo (probetas de $20 \times 20 \times 2,5 \mathrm{~cm}$ ).

Ensayos de campo

-Ensayo de intemperie (probetas de $20 \times 20 \times 2,5 \mathrm{~cm}$ ). 
- Ensayo de lluvia artificial (muros de $50 \mathrm{~cm}$ de espesor x $1 \mathrm{~m}$ de largo y $1 \mathrm{~m}$ de alto).

Los ensayos de laboratorio se han realizado en las instalaciones del Departamento en la Escuela Técnica Superior de Arquitectura y la fabricación de probetas y

TABLA 1

\begin{tabular}{|c|c|c|c|c|}
\hline \multicolumn{2}{|c|}{ Aditivo } & Mezcla & Proporción & $\begin{array}{c}\mathrm{N}^{\circ} \\
\text { Identif. }\end{array}$ \\
\hline \multirow{3}{*}{\multicolumn{2}{|c|}{ Sikacem }} & \multirow{3}{*}{ (Vol. agua) } & $1: 2$ & 11 \\
\hline & & & $1: 7$ & 12 \\
\hline & & & $1: 15$ & 13 \\
\hline \multirow{3}{*}{\multicolumn{2}{|c|}{ Sikalatex }} & \multirow[t]{3}{*}{ (Vol. agua) } & $1: 2$ & 21 \\
\hline & & & $1: 15$ & 22 \\
\hline & & & $1: 99$ & 23 \\
\hline \multirow{3}{*}{\multicolumn{2}{|c|}{ Linaza }} & \multirow[t]{3}{*}{ (Vol. agua) } & $1: 99$ & 31 \\
\hline & & & $1: 15$ & 32 \\
\hline & & & $1: 9$ & 33 \\
\hline \multirow{3}{*}{\multicolumn{2}{|c|}{ Sika 1}} & \multirow[t]{3}{*}{ (Vol. agua) } & $1: 9$ & 41 \\
\hline & & & $1: 15$ & 42 \\
\hline & & & $1: 99$ & 43 \\
\hline \multirow{3}{*}{\multicolumn{2}{|c|}{ Sikalite }} & \multirow[t]{3}{*}{ ( Vol. tierra) } & $1 \%$ & 51 \\
\hline & & & $2 \%$ & 52 \\
\hline & & & $3 \%$ & 53 \\
\hline \multirow{3}{*}{$\begin{array}{c}\text { Azufre } \\
+ \\
\text { cal } \\
\end{array}$} & $1: 3$ & \multirow[t]{3}{*}{ ( Vol. tierra) } & \multirow{3}{*}{$7,7 \%$} & 61 \\
\hline & $1: 1$ & & & 62 \\
\hline & $1: 2$ & & & 63 \\
\hline $\mathrm{Cal}$ & $1: 1$ & \multirow[t]{3}{*}{ (Vol. tierra) } & $2 \%$ & 71 \\
\hline \multirow{2}{*}{$\begin{array}{c}+ \\
\text { cenizas }\end{array}$} & $1: 2$ & & $10 \%$ & 72 \\
\hline & $1: 3$ & & $20 \%$ & 73 \\
\hline \multirow{3}{*}{\multicolumn{2}{|c|}{ Yeso }} & \multirow[t]{3}{*}{ (Vol. tierra) } & $1: 15$ & 81 \\
\hline & & & $1: 12$ & 82 \\
\hline & & & $1: 9$ & 83 \\
\hline \multirow{3}{*}{\multicolumn{2}{|c|}{ Estabiram }} & \multirow[t]{3}{*}{ (Vol agua) } & $0,07 \%$ & 91 \\
\hline & & & $0,11 \%$ & 92 \\
\hline & & & $1,7 \%$ & 93 \\
\hline \multirow{3}{*}{\multicolumn{2}{|c|}{ Dodigen }} & \multirow[t]{3}{*}{ (Vol. agua) } & $0,07 \%$ & 101 \\
\hline & & & $0,11 \%$ & 102 \\
\hline & & & $1,7 \%$ & 103 \\
\hline & & (Vol agua) & $0,07 \%$ & 111 \\
\hline Deterge & & & $0,11 \%$ & 112 \\
\hline & & & $1,7 \%$ & 113 \\
\hline Aceite de & oliva & (Vol. agua) & $1: 99$ & 121 \\
\hline & & & $1: 15$ & 122 \\
\hline & & & $1: 9$ & 123 \\
\hline Cal & $2: 3$ & ( Vol. tierra) & & 131 \\
\hline+ & $4: 1$ & & $4,00 \%$ & 132 \\
\hline cemento & $1: 1$ & & & 133 \\
\hline Ceme & & ( Vol. tierra) & $4 \%$ & 141 \\
\hline & & & $2 \%$ & 142 \\
\hline & & & $6 \%$ & 143 \\
\hline $\mathrm{Cal}$ & & ( Vol. tierra) & $1: 15$ & 151 \\
\hline & & & $1: 12$ & 152. \\
\hline & & & $1: 9$ & 153 \\
\hline Agua de & $\mathrm{cal}$ & (Vol. agua) & $5 \%$ & 161 \\
\hline & & & $7,5 \%$ & 162 \\
\hline & & & $10 \%$ & -163 \\
\hline
\end{tabular}

construcción de muros, así como los ensayos a la intemperie, en el centro experimental de "Inter-acción", en la localidad de Navapalos (Soria).

Los productos hidrofugantes elegidos pertenecen, en su mayoría, a la marca SIKA, por su gran difusión y accesibilidad, aunque también se han ensayado otros productos comerciales de menor aplicación en la construcción, como el azufre o el aceite de oliva y otras sustancias menos comerciales comoel Dodigen o Estabiram, derivados del petróleo, así como los más tradicionales: cemento, cal y yeso (Tabla 1). Se utilizaron un total de 16 mezclas con 3 dosificaciones distintas, una dosificación según las indicaciones del fabricante para el uso habitual de del producto y otras dos, en mayor y menor proporción respectivamente, ensayando 3 probetas iguales por cada dosificación (lo que hace un total de 144 probetas por ensayo).

Debido a la inexistencia de una norma específica para este material, las pruebas de absorción y heladicidad se han intentado ajustar a las normas ASTM (D-557, Wetting and Drying Test y D-560, Freezing and Thawing Test) para suelo-cemento compactado (5). Estos ensayos tienen por objeto determinar la pérdida de material, cambios de humedady volumen producidos por repetidosy alternativos ciclos, que intentan reproducir de manera acelerada el comportamiento del material en condiciones extremas (Figs.7 y 8). Para el ensayo de goteo se dispusieron las baldosas en un soporte inclinado $45^{\circ}$ sobre el suelo y, a una altura de $2 \mathrm{~m}$, se colocó un recipiente lleno de agua y un grifo que regula la salida de la misma hasta obtener una gota por segundo, manteniendo el proceso durante 12 horas.

El ensayo de lluvia artifícial se realizó disponiendo 22 muros, en dos círculos de $5 \mathrm{~m}$ de diámetro y colocando un aspersor en el centro (6), de manera que el agua incidiera directamente a media altura de las caras interiores (Fig.9), midiendo la profundidad del surco originado por

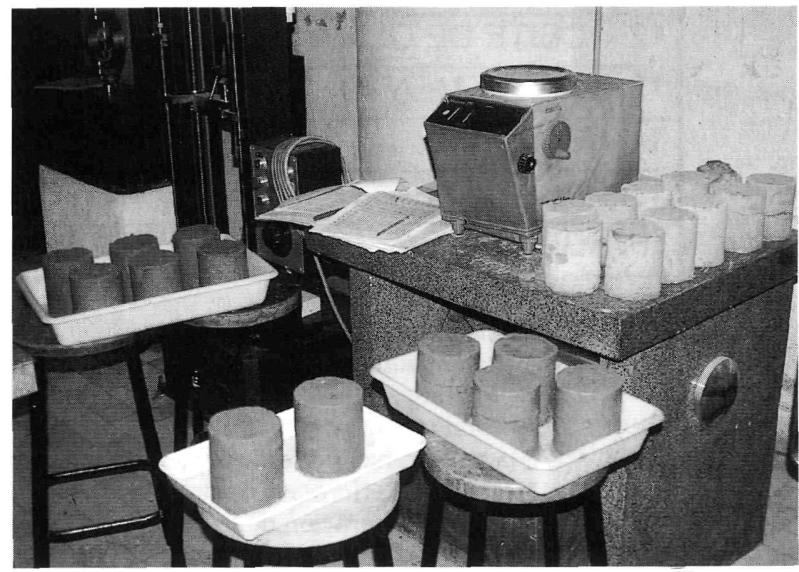

Fig. 7.- Probetas sometidas a ciclos de hielo-deshielo. Laboratorio de Materiales del DCTA. 


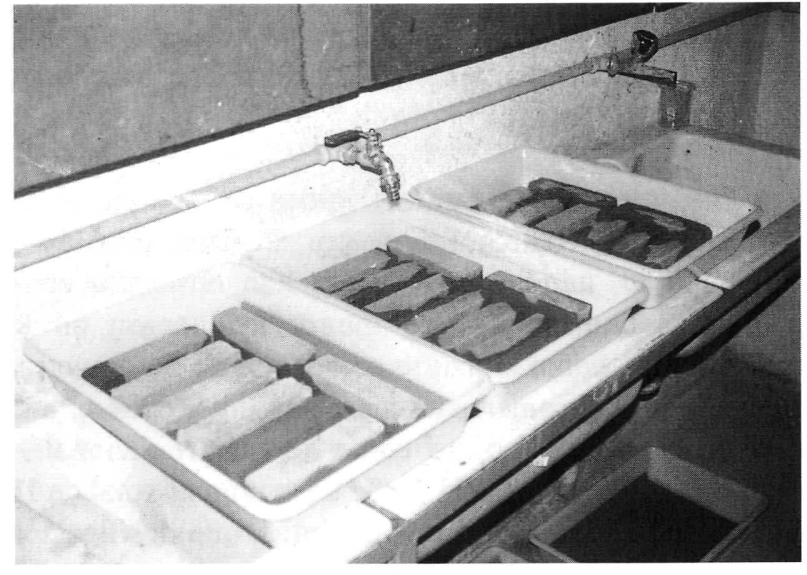

Fig. 8.- Probetas sometidas a humedecimiento en uno de los ciclos de ensayo. Laboratorio de Materiales del DCTA.

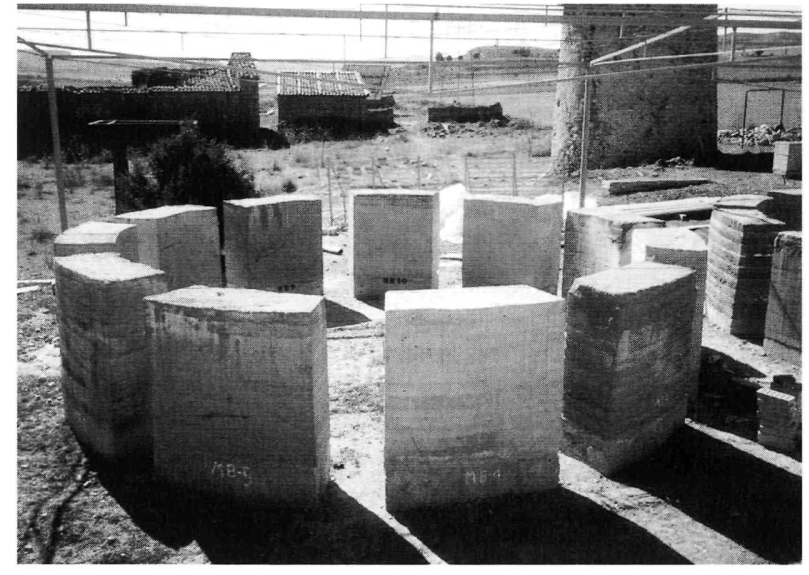

Fig. 9.- Disposición de los muros en uno de los círculos para el ensayo de lluvia artificial. Centro Navapalos.

TABLA 2

RESULTADOS DE ENSAYOS DE COMPORTAMIENTO FRENTE AL AGUA

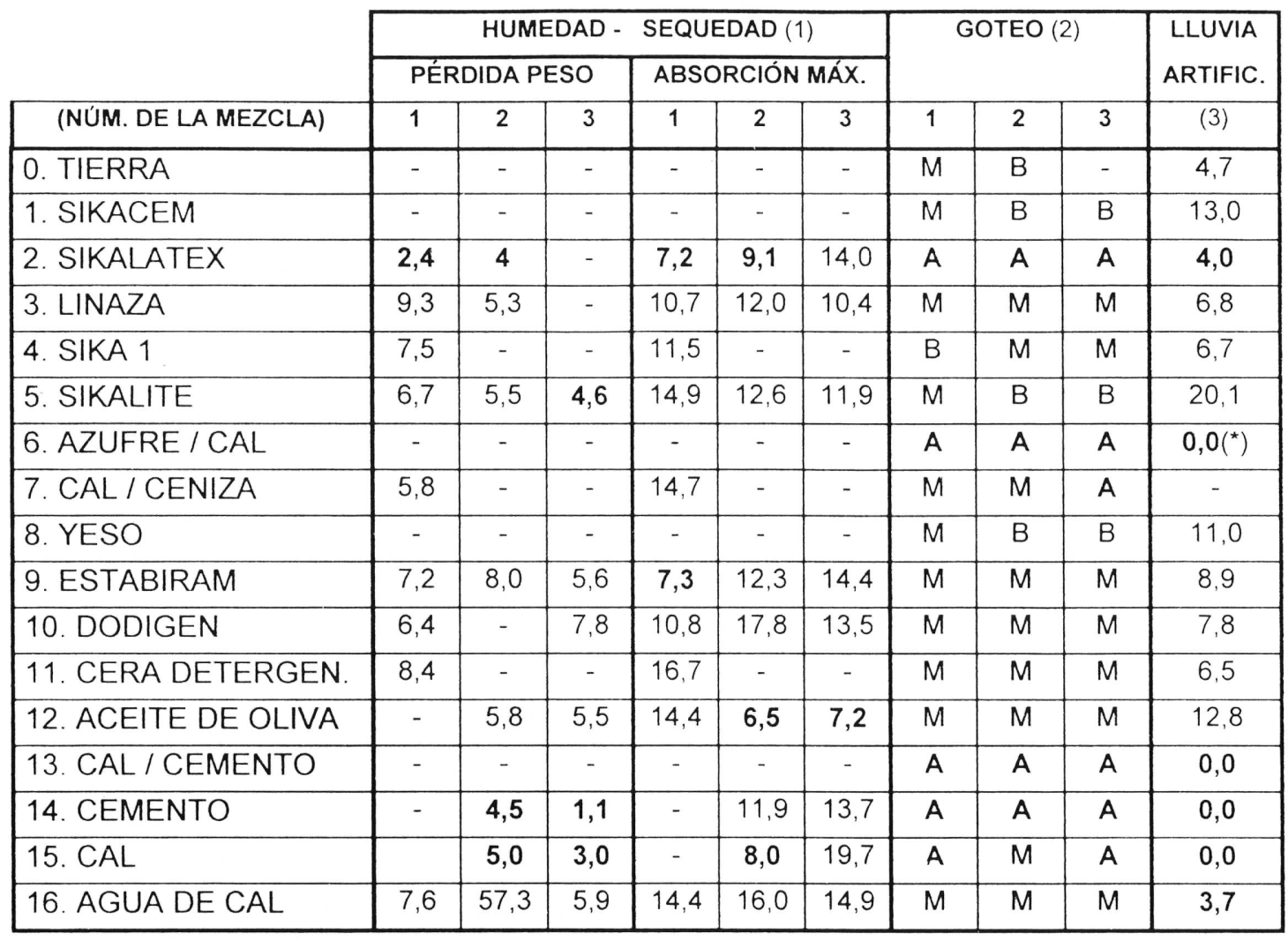

(1) Valores en \% de peso inicial

(-) No han superado los 12 ciclos del ensayo

(2) Comportamiento del material:

A. (Alto): No hay perforaciones. Ligero escurrimiento

M. (Medio): Perforación hasta $1 \mathrm{~cm}$. Reblandecimiento

B. (Bajo): Perforación de más de $1 \mathrm{~cm}$. Desprendimientos

(3) Profundidad de la erosión en $\mathrm{cm}$

(*) Minima erosión superficial 
el agua. Para la fabricación de los muros se establecieron cuatro tipos de protección: en un primer grupo, se consideraron estabilizantes que se incorporaban a la tierra en el proceso de preparación de la misma; en un segundo grupo se trataron estabilizantes que se incorporaban al agua con que ésta se humedecía; en un tercer grupo se englobaban los productos de aplicación superficial y, en el cuarto, la protección se proporcionaba a través del procedimiento constructivo tradicional (calicostrado) (Tablas 2 y 3 ).

\subsection{Conclusiones}

Del estudio documental realizado se concluye que, a pesar del vasto patrimonio existente en la península, son escasos los textos específicos en lengua castellana y se echa en falta una red de información organizada a nivel nacional que permita el conocimiento de las actividades que se dan, de modo disperso, en el ámbito de la conservación del Patrimonio construido con tierra. La búsqueda de información en los países vecinos, Francia y Portugal, ha resultado más fructífera, consiguiéndose contactar con varias organizaciones que desarrollan programas regionales de recuperación y conservación del Patrimonio
Arquitectónico construido con tierra, de gran interés para el estudio que nos ocupa.

En cuanto a la técnica del tapial, en esta última década se han publicado en España tan sólo dos monografias (ambas recogidas en la bibliografía). Respecto a este tema, la tesis doctoral de Mariano Olcese (7) recoge un amplio estudio, desde los tratados de arquitectura a nuestro siglo, pudiéndose corroborar cómo hasta la década de los setenta no aparecen las escasas publicaciones existentes, hasta entonces se pueden encontrar algunos artículos y referencias en obras de mayor amplitud sobre arquitectura popular, en general. En lo tocante a intervenciones en el Patrimonio, encontramos varias referencias en las actas de diversos congresos y jornadas realizados en los últimos diez años, todas ellas pertenecientes a intervenciones en fortificaciones o recintos amurallados, dejándose de lado aquello que podriamos describir como patrimonio no catalogado o arquitecturas no históricas.

La información recogida en la mayoría de los textos sobre arquitectura popular es de carácter general y poco precisa en lo que a procesos constructivos se refiere. El abandono de la práctica de este sistema constructivo en nuestra

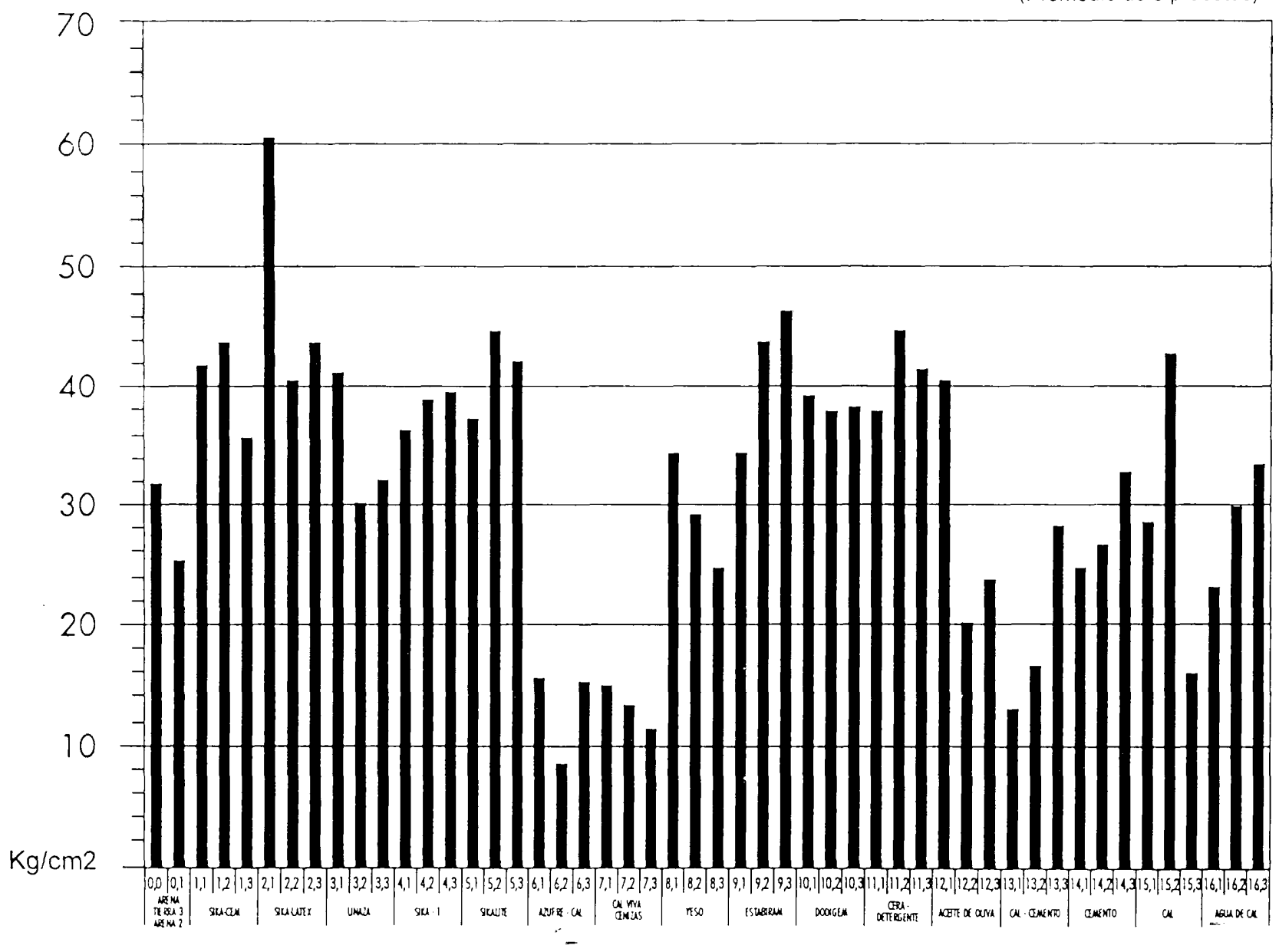


región, desde mediados de siglo, ha conducido al desconocimiento absoluto, hoy en día, de muchas de las dificultades de su puesta en obra, por lo que resulta imprescindible el desarrollo de programas de formación específica de operarios en este tipo de técnicas, actualmente inexistentes.

A la vista de los análisis realizados y de los resultados obtenidos en los ensayos, podemos sacar las siguientes conclusiones:

1. Del estudio de campo y documental se desprende que la causa más común de los daños en fábricas de tierra (tapiales) es la presencia de agua, mientras que la resistencia mecánica nunca es un problema, ya que tradicionalmente las dimensiones responden a criterios de estabilidad y sistema de ejecución, siendo muy escasa la presencia de daños debidos a exceso de solicitación of falta de resistencia. En este sentido, resultan más significativos los resultados de los ensayos que estudian el comportamiento de la tierra ante el agua (humedad/sequedad, hielo/deshielo, goteo, lluvia artificial) que a aquéllos referidos a resistencia o dureza superficial.

2. Es posible la aplicación de determinados productos comerciales existentes para mejorar el comportamiento de los muros de tapial, aunque estos productos no se fabricaran inicialmente para este fin, puesto que se trata, principalmente, de aditivos para morteros y hormigones.

3. Para mejorar la resistencia mecánica de los muros de tapial es necesario mezclar estos productos con la tierra, antes de su compactación. Sin embargo, para disminuir la erosión hídrica y la absorción de agua, en intervenciones sobre el Patrimonio construido con tierra, la técnica más adecuada será la aplicación de tratamientos superficiales en sucesivas capas que garanticen la penetración adecuada. En los ensayos se observaron diferencias significativas entre muros tratados con un sola capa o varias.

4. Los resultados conseguidos con diferentes productos comerciales (en concreto los de la casa SIKA), son descritos a continuación. Debe advertirse, sin embargo, que estas mejoras deberían ser evaluadas a lo largo del tiempo antes de aplicarlas a la recuperación de fábricas de tierra pertenecientes al Patrimonio.

. El producto que mejora en mayor grado los muros de tierra es el SIKALATEX, que añadido al agua -utilizada para humedecer la tierra, en proporción 1 parte de SIKALATEX por 15 partes de agua-, disminuye la absorción de la misma y la erosión hídrica, a la vez que aumenta la resistencia a compresión y la dureza superficial. Aunque los resultados obtenidos en conjunto no superan a los que se dan con otros materiales tradicionales (cal o cemento), el comportamiento en muros y probetas presenta mayor homogeneidad. E1
. EL DODIGEN, mezclado de la misma manera que el anterior, en la proporción de 1 parte de DODIGEN por 9 partes de agua, mejora la resistencia a compresión y la dureza superficial, pero su comportamiento frente al agua es algo peor que el del SIKALATEX.

. Los demás productos ensayados: SIKACEM, SIKA 1, SIKALITE y ESTABIRAM, mejoran la resistencia a compresión, pero presentan, en general, una erosión hídrica muy acusada y no disminuyen la absorción de agua, por lo que se consideran poco adecuados para mejorar los muros de tapial.

5. De los productos tradicionales que se han ensayado para mejorar los muros de tierra, se han obtenido los siguientes resultados:

. La cal y el cemento, así como las mezclas de ambos, añadidas a la tierra antes de su compactación, mejoran la resistencia a compresión, la dureza superficial y la erosión hídrica en los muros, pero el grado de absorción de agua en los ensayos de laboratorio ha sido muy variable. Se debe prestar especial atención a este factor en el proceso de preparación del material para obtener unos resultados satisfactorios, especialmente en aquellas zonas donde el agua se manifieste, climatológicamente, de forma relevante.

- Las mezclas de cal con otros productos (cal y azufre, cal y cenizas), dan como resultado un empeoramiento de la resistencia a compresión, además de aumentar la absorción de agua, por lo que los consideramos no adecuados para mejorar los muros de tierra.

- El yeso dispara los valores de absorción de agua, por lo que se considera un producto no adecuado.

- La adición de aceites (de oliva, de linaza), disminuye algo la absorción de agua, pero no la erosión hídrica. No se aprecian cambios substanciales en la resistencia a compresión. Los efectos de estos productos no son muy acusados, considerándose que no son adecuados para mejorar el comportamiento de los muros de tapial.

De cara al futuro proponemos las siguientes líneas de trabajo como continuación de las investigaciones:

1. Construcción de modelos, a escala real, para evaluar en ellos la validez de las conclusiones obtenidas de la experimentación con probetas y la evolución, a lo largo del tiempo, de los productos aplicados.

2. Desarrollo de una normativa específica sobre la tierra como material de construcción (8)._Para la elaboración de esta normativa se propone desarrollar los siguientes aspectos: 
. Obtención de parámetros que determinen la idoneidad de los productos comerciales aplicados, desarrollando con más detalle alguno de los ensayos realizados.

.Obtención de equivalencias entre los resultados de laboratorio y el comportamiento de modelos, a escala real, como ya se ha indicado en el punto anterior.
3. Establecer líneas de cooperación con las casas comerciales cuyos productos se ha concluido que son adecuados para el desarrollo de técnicas de intervención sobre patrimonio arquitectónico construido con muros de tapial, con el fin de ajustar convenientemente dichos productos a su comercialización con este nuevo uso.

\section{BIBLIOGRAFIA}

- KEABLE, JULIAN: (1996). Rammed Earth Structures. A Code of Practice. Intermediate Technology Publications. London (U.K.)

- BAULUZ DEL RÍO, GONZALO: (1992). Bases para el Diseño y Construcción con Tapial. Ministerio de Obras Públicas y Transportes. Madrid (España)

- FONT, F. HIDALGO, P: (1991). El Tapial. Una técnica de construcción milenaria. Colegio Oficial de Aparejadores de Castellón. (España)
- INSTITUTOEDUARDOTORROJA: (1987). Latierra material de construcción. Monografia ${ }^{\circ}$ 385-386. Madrid (España)

- GRAHAM MCHENRY, PAUL: (1984). Adobe and Rammed Earth Buildings. Design and Construction. The university of Arizona Press. Tucson (New Mexico, U.S.A.)

- MIDDLETON, G.F.: (1954, $4^{\text {th }}$ ed reprinted 1995). Earth wall Construction. Division of Building, Construction and Engineering. Sidney (Australia)

\section{NOTAS}

(1) Para mayor información sobre la distribución geográfica de las técnicas de construcción con tierra en Madrid puede consultarse: Rohmer, E. (1987) Las construcciones en tierra: Adobes y tapiales en la Comunidad de Madrid. Ponencia recogida en las actas del ler encuentro detrabajo Navapalos 86. Publicado por el servicio de agricultura de la Diputación Provincial de Soria.

(2) La documentación resultante de este trabajo se encuentra archivada en el Departamento de Construcción y Tecnología arquitectónicas de la E.T.S.A.M. a disposición de todas aquellas personas interesadas.

(3) Tradicionalmente la palabra tapial se refiere al propio encofrado utilizado en la construcción y, por extensión, a la técnica constructiva e incluso, a la propia fábrica, confundiéndose con tapia.

(4) Brenca. Vocablo de origen catalàn que se refiere a la media luna de material más resistente que se forma en cada tramo de las tapias reforzadas, de ahi "tapia de brencas". Esta técnica es muy común en la submeseta sur y es la más característica del área meridional de la Comunidad de Madrid. En algunos dialectos se utiliza para designar las jambas de puertas y ventanas.

(5) NLT-302/72 del Laboratorio de Transporte y Mecánica del suelo "José Luis Escario" (versión en castellano).

(6) El aspersor completa una vuelta cada 7 segundos y el riego se produce durante una hora, dos veces por día, con un intervalo de 10 horas, hasta completar 10 ciclos. El agua se proyecta con una presión aproximada de $4 \mathrm{~kg} / \mathrm{cm}^{2}$.

(7) Olcese Segarra, Mariano (1993). Arquitecturas de tierra: Tapial y adobe. Colegio Oficial de Arquitectos de Valladolid.

(8) Es interesante tomar como referencia el trabajo iniciado por el grupo "Habiterra" (Red temática XIV.Adel programa CYTED: Sistematización del uso del tierra enviviendas de interés social), con la publicación de las Recomendaciones para la elaboración de normas técnicas de edificación con adobe, tapial, ladrillos y bloques de suelo-cemento (La Paz, 1995) 